\title{
Ultrasound in the diagnosis of anatomical variation of anterior and posterior tibial arteries.
}

\author{
Miao Zheng, Chuang Chen, Qianyi Qiu, Changjun Wu
}

Department of Ultrasound, the First Affliated Hospital of Harbin Medical University, Harbin, Heilongjiang, China

\begin{abstract}
Aims: Knowledge about branching pattern of the popliteal artery is very important in any clinical settings involving the anterior and posterior tibial arteries. This study aims to elucidate the anatomical variation patterns and common types of anterior tibial artery (ATA) and posterior tibial arteries (PTA) in the general population in China. Material and methods: Anatomical variations of ATA, PTA, and peroneal artery were evaluated with ultrasound in a total of 942 lower extremity arteries in 471 patients. Results: Three patterns of course in the PTA were ultrasonographically identified: 1) PTA1: normal anatomy with posterior tibial artery entering tarsal tunnel to perfuse the foot $(91.5 \%)$, 2) PTA2: tibial artery agenetic, and replaced by communicating branches of peroneal artery entering tarsal tunnel above the medial malleolus to perfuse the foot (5.9\%), and 3) PTA3: hypoplastic or aplastic posterior tibial artery communicating above the medial malleolus with thick branches of peroneal artery to form a common trunk entering into the tarsal tunnel (2.4\%). In cases where ATA was hypoplastic or aplastic, thick branches of the peroneal artery replaced the anterior tibial artery to give rise to dorsalis pedis artery, with a total incidence of $3.2 \%$ in patients, and were observed more commonly in females than in males. Hypoplastic or aplastic termini of ATA and PTA, with perfusion of the foot solely by the peroneal artery, was identified in 1 case. In another case, both communicating branches of the peroneal artery and PTA entered the tarsal tunnel to form lateral and medial plantar arteries.

Conclusions: Anatomical variation of ATA and PTA is relatively common in the normal population. Caution should be exercised with these variations when preparing a peroneal artery vascular pedicle flap grafting. Ultrasound evaluation provides accurate and reliable information on the variations.
\end{abstract}

Keywords: posterior tibial artery, anterior tibial artery, peroneal artery, variation, ultrasound

\section{Introduction}

The popliteal artery provides not only perfusion to capsule and ligaments of the knee joint via its genicular branches, but also the majority blood supply for the lower extremity [1-5]. Continuing from the femoral artery and passing through the popliteal fossa, it normally splits at the lower border of the popliteus muscle in the anterior and posterior tibial arteries. The anterior tibial ar-

Received 25.08.2015 Accepted 10.10.2015

Med Ultrason

2016, Vol. 18, No 1, 64-69

Corresponding author: Changjun Wu, M.D.

Department of Ultrasound,

the First Affliated Hospital

of Harbin Medical University,

No. 23 Youzheng Street,

Harbin, Heilongjiang, 150001, China

Phone: +8613351100162

Fax: +86045185556000

E-mail: cjwcdh@163.com terymost commonly passes between the tibia and fibula, then moves inferiorly down the leg after passing through a gap in the interosseous membrane, and runs down the entire length of the leg into the foot, ending at the dorsalis pedis artery. The posterior tibial artery, usually the larger of the two popliteal artery branches, continues from the popliteal artery along the surface of the tibialis posterior. Accompanied by the tibial nerve, it descends beneath the calf muscle by penetrating the lateral compartment of the leg and enters the sole of the foot via the tarsal tunnel. Normally, the peroneal artery, also known as the fibular artery, arises from the tibial-fibular trunk just below the knee. It supplies the deep posterior, lateral and anterior compartments of the leg and the fibula.

Due to embryological abnormalities of the arterial pattern, variations in anatomy of the lower limb arterial network, such as absence, rudimentary reminders, variant courses and replacement areas of vascularization, are statistically common [6-8]. These variant arterial 
supplies to foot and/or leg are of important anatomical significance and great clinical relevance, especially in plastic and vascular surgeries in an effort to reconstructing lower limb arteries or preparing viable muscle flaps [9-13]. The knowledge of these variations will definitely help reduce the risk of vascular trauma and unnecessary haemorrhage during lower limb surgery.

Anatomical variations of the popliteal artery have been extensively studied $[1,9,10,12,14,15]$ with an impact on the daily clinical practice in the field of surgery and during catheterization. Studies have shown that the popliteal artery can trifurcate, gives high division or hypoplastic-aplastic branches with altered distal supply. In fact, hypoplastic changes have been reported for the anterior tibial, posterior tibial, and peroneal arteries $[1,2,9,10,12,14-24]$. The information about the anatomy of limb arteries is usually obtained via ultrasonography, computer tomography angiography (CTA), magnetic resonance angiography (MRA), and/or anatomical dissection. CTA and MRA, most performed pre-operatively, provide adequate radiological information about the studied vasculature, but require contrast agents and expensive equipment and are labor-intensive and timeconsuming. Anatomical dissection, on the other hand, yields thorough and accurate anatomical information of the investigated structure, but is often limited by the lack of highly trained professionals and specimens. Vascular ultrasonography, with the advantage of noninvasiveness, and low-cost has gained increasing popularity in the examination of lower extremity vascularity. It is especially valuable in the diagnosis of vascular disease in a community hospital setting.

Herein we report results of anatomical variations of the posterior tibial artery, anterior tibial artery and peroneal artery, from 942 limbs in 471 subjects by using this method. Three patterns of course in the posterior tibial artery were identified.

\section{Materials and Methods}

\section{Patients}

A total of 942 lower extremity arteries in 471 patients (male 226) between ages of 17 to 87 years (average 43.9 years), were examined for lower limb vessels from June 2014 to October 2014 with ultrasound, after excluding cases with indiscernible anterior tibial artery (ATA), posterior tibial artery (PTA), and peroneal artery due to limb swelling, lower extremity arterial occlusive/arteriosclerosis, limb ulcers, skin diseases, and other reasons.

\section{Ultrasound examination}

ATA, PTA, and peroneal artery were imaged up to the bifurcation of popliteal artery with the patients in supine and prone position using Duplex ultrasound (Sequoia 512, SIEMENS Medical Systems) with 4C1 and 8L5 probes.

When the atherosclerotic arteries appeared with deflated occlusion with a diameter less than $1 \mathrm{~mm}$, and these patients presented ankle and foot skin lesions and tissue edema, ultrasound examination of the bifurcating branches of the peroneal artery at the junction site to the peroneal artery was limited by unsatisfactory resolution, and the atherosclerotic arteries were excluded from the study.

Arterial aplasia was defined as no artery detected after ruling out the presence of arteriosclerosis obliterans, skin lesions, excessive tissue edema, and other limiting factors for imaging. Arterial hypoplasia was defined as a smooth, small artery with a diameter less than $1 \mathrm{~mm}$, ruling out arterial narrowing secondary to other conditions.

The ultrasound examination was carried out by 2 radiologists specialized in vascular ultrasonography and with an expertise of vascular anatomy of lower extremity and ultrasound imaging.

\section{Statistical analysis}

Data were expressed as frequency and percentage (\%), statistical differences of percentage between two groups were determined by Chi-square test. SPSS 16.0 software was used for statistical analysis. $\mathrm{p}<0.05$ was considered statistically significant.

\section{Results}

In the 471 patients, the relationship of the posterior tibial artery and peroneal artery had three patterns:

1) PTA1 (normal anatomy): The peroneal artery runs medial to the fibula, terminates at the lateral ankle as medial and lateral malleolar branches merging into the anterior and posterior tibial arteries. While the PTA runs between the soleus and deep calf muscle, entering tar-

Table I. Variation of anterior (ATA) and posterior tibial arteries (PTA)

\begin{tabular}{llll}
\hline Variation & $\begin{array}{l}\text { Unilateral } \\
\mathrm{n}=48(\%)\end{array}$ & $\begin{array}{l}\text { Bilateral } \\
\mathrm{n}=31(\%)\end{array}$ & $\begin{array}{l}\text { Total } \\
\mathrm{n}=471(\%)\end{array}$ \\
\hline PTA & & & \\
$\quad$ PTA2 & $16(33.3)$ & $19(61.3)$ & $\begin{array}{l}35(74.3) \\
18(3.8)\end{array}$ \\
PTA3 & $14(29.2)$ & $4(12.9)$ & \\
ATA & & & $22(4.7)$ \\
$\quad$ ATA2 & $16(33.3)$ & $6(19.4)$ & $4(0.8)$ \\
$\begin{array}{l}\text { Other variation } \\
\text { Total }\end{array}$ & $2(4.2) *$ & $2(6.5)^{\Delta}$ & $392(83.3)$ \\
\hline
\end{tabular}

*Two other patterns of variation were also observed in two cases (see the main text). ${ }^{\Delta}$ Different variations in the bilateral limbs. In one case, right PTA2 and left PTA3; In the other case, left PTA2 and right ATA2; $\mathrm{n}$ - number of patients 
sal tunnel to form the medial and lateral plantar arteries, communicating with small branches of the peroneal artery right above the medial malleolar. These normal anatomies of the lower extremity arteries were diagnosed in 414 of 471 patients $(87.9 \%)$.

2) PTA2: Hypoplastic or aplastic trunk of PTA disappeared before the tarsal tunnel. Thick communicating branches from the peroneal artery enter the tarsal tunnel to provide blood supply to the foot (fig 1a). Medial deviation of the trunk of the peroneal artery was observed in 35 of 471 patients (7.4\%), including 3.4\% (16/471) patients with the unilateral variation, and $4.0 \%(19 / 471)$ with bilateral variation (as shown in Table I).

3) PTA3: Hypoplastic or aplastic PTA entered the tarsal tunnel with branches from the thick peroneal artery in a common trunk, while perforation branches of the peroneal artery provided a blood supply for the plantar. The cases with the diameter of communicating branches of the peroneal artery no less than that of posterior tibial artery on the same level were assigned to this category (fig 1b). This pattern had an incidence of 3.8\% (18/471) patients, including $3.0 \%(14 / 471)$ patients with the unilateral variation, and $0.8 \%(4 / 471)$ with bilateral variation (shown in Table I).

No differences of frequency distribution between the lower extremity arteries of male and female with PTA1, PTA2 and PTA3 were observed ( $>>0.05)$, and no differences were also observed between left and right lower extremity arteries ( $>0.05)$, as shown in Table II.
Overall, $392(83.3 \%)$ of the subjects in this study had a normal three branching pattern of the popliteal artery, a pattern described previously.

The course of the ATA was revealed in two patterns:

1) ATA1 (normal anatomy): After departing from the popliteal artery, the ATA penetrated the interosseous membrane to reach the lateral anterior aspect of the tibia, and became dorsalis pedis in the midpoint between the medial and lateral malleolars. This normal anatomy was found in 447 of 471 patients $(94.9 \%)$.

2) ATA2: In 22 of 471 (4.7\%) patients (16 patients with unilateral and 6 patients with bilateral) ATAs were with fine termini after giving rise to muscle and cutaneous branches. The thick peroneal artery communicated with or even replaced terminal ATAs (fig 1c). In such variations, the dorsalis pedis arteries usually were located medial to the malleolus. Lower extremity arteries with ATA2 were observed more commonly in females than in males (female 5.1\%, male $1.1 \%$, $\mathrm{p}<0.001$ ), and no differences were observed between lower extremity arteries of left and right, data were showed in Table III.

In two $(0.4 \%)$ patients other patterns of variation were observed. In one case, both ATA and PTA were absent, with communicating branches from the peroneal artery supplying the foot. In the other case, the thin trunk of PTA entered the tarsal tunnel with a communicating branch from the peroneal artery (fig 1d), both were palpable in the tarsal tunnel.

Table II. Distributary types of posterior tibial arteries (PTA)

\begin{tabular}{|c|c|c|c|c|c|c|}
\hline & \multirow{2}{*}{$\begin{array}{l}\text { Frequency of } \\
\text { limb }\end{array}$} & \multirow[b]{2}{*}{ PTA1 } & \multicolumn{2}{|c|}{ Type (\%) } & \multirow[t]{2}{*}{ Other variation } & \multirow[t]{2}{*}{ p } \\
\hline & & & PTA2 & PTA3 & & \\
\hline Male & $452(48.0)$ & $417(48.4)$ & $28(50.0)$ & $7(30.4)$ & & \multirow{2}{*}{$>0.05$} \\
\hline Female & $490(52.0)$ & $444(51.6)$ & $28(50.0)$ & $16(69.6)$ & $2(100)^{\Delta *}$ & \\
\hline Left & $471(50.0)$ & $425(49.4)$ & $30(53.6)$ & $15(65.2)$ & $1(50)^{\Delta}$ & \multirow{3}{*}{0.05} \\
\hline Right & $471(50.0)$ & $436(50.6)$ & $26(46.4)$ & $8(34.8)$ & $1(50)^{*}$ & \\
\hline Total $(\%)$ & 942 & $861(91.5)$ & $56(5.9)$ & $23(2.4)$ & $2(0.2)$ & \\
\hline
\end{tabular}

${ }^{\Delta}$ On the left side of the ankle canal, branch of peroneal artery and the posterior tibial artery coexist; *The right side of the peroneal artery branch replaced the pretibial artery and posterior tibial artery

Table III. Distributary types of ATA

\begin{tabular}{lllll}
\hline & Frequency of limb & Type (\%) & Other variation & P \\
& & ATA1 & ATA2 & \\
\hline Male & $452(48.0)$ & $446(49.0)$ & $5(16.7)$ & \\
Female & $490(52.0)$ & $465(51.0)$ & $25(83.3)$ & $1(100)^{*}$ \\
Left & $471(50.0)$ & $456(50.0)$ & $14(46.7)$ & \\
Right & $471(50.0)$ & $455(50.0)$ & $16(53.3)$ & $1(100)^{*}$ \\
Total(\%) & 942 & $911(96.7)$ & $30(3.2)$ & $1(0.1)$ \\
\hline
\end{tabular}

*The right branch of the peroneal artery replaced the pretibial artery and posterior tibial artery 


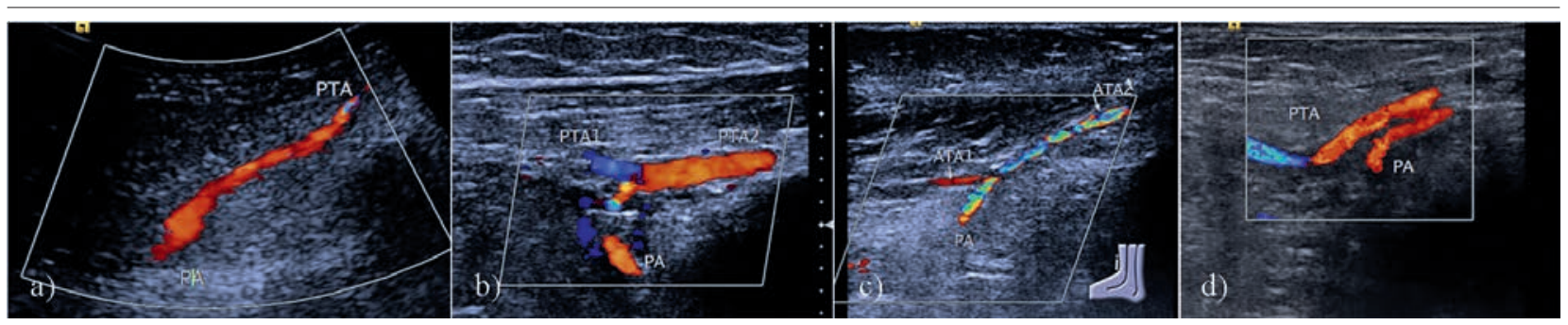

Fig 1. a) Peroneal artery (PA) completely replaced posterior tibial artery (PTA) forward lined into the ankle tube, the ramus communicans went straight; b) Ramus communicans of the Peroneal artery (PA) and the endpiece of the posterior tibial artery (PTA1) merged into the ankle district of the posterior tibial artery (PTA2); c) End piece of the anterior tibial artery (ATA2) converged by the anterior tibial artery (ATA1) and ramus communicans of the peroneal artery(PA); c) Two arteries in the ankle district, the upper one was the posterior tibial artery (PTA), the one below was the ramus communicans of the proneal artery (PA)

\section{Discussions}

The lower limb arteries are derived from an embryonic vessel that supplies the lower limbs, namely the primitive middle and distal segments of the sciatic artery [25-27].

Normally, after starting at the edge of the popliteal muscle, the PTA normally passes between the shallow and deep muscle groups, enters into the tarsal tunnel on the medial edge of the Achilles tendon to divide into plantar and lateral arteries. The peroneal artery usually starts slightly below the very beginning part of the posterior tibial artery, descends along the inner side of the fibula, ending at the rear of the lateral malleolus rami, with small communicating branches connecting anterior and posterior arteries at the level of 3 to $8 \mathrm{~cm}$ above ankle. However, the prevalence of anatomic variability in the lower limb arteries is common due to persistent primitive arterial segments, abnormal fusions, segmental hypoplasia, or the absence of these arteries.

Lippert et al classified the anatomical variation of the popliteal artery in males according to the starting position of the popliteal artery, anterior tibial artery, posterior tibial artery and the peroneal [28]. Kim et al modified Lippert's classification, and described the third variation of the popliteal artery [29]. In the population studied, we found that $83.3 \%$ of the individuals presented a normal three branching pattern of the popliteal artery.

Arterial anatomies of lower limb have great radiological and surgical importance. Reportedly, the variation rate can be as high as $13 \%[1,9,21,30,31]$, which necessitates thorough examination of the vascular anatomy in the lower extremity prior to any intervention. Without a precise knowledge of anatomic variations in the branching pattern of the popliteal artery, it can be limb-threatening during a number of procedures in the lower limb, including arterial reconstructions in femoro-distal bypass graft procedures, embolectomy, and catheterization to treat lower limb ischemia in diabetic and ankle arthroscopy.

In the present study, economic and convenient ultrasonography was chosen to visualize lower limb vasculature. By virtue of ultrasound vascular examination, we identified 3 different patterns of course of PTA, described above. The incidences of PTA1, PTA2 and PTA3 in the studied population were $91.5 \%, 5.9 \%$, and $2.4 \%$, respectively. We also found that under circumstances of hypoplastic or aplastic ATA, or hypoplastic or aplastic PTA, the thick communicating branches or truck of the peroneal artery merged into or even replaced the narrowed anterior or posterior tibial artery to secure plantar arterial blood supply. The incidence of replacement of the PTA by the peroneal artery was $5.9 \%$, consistent with the reported incidence of $3.8 \%$ to $5.1 \%$ on angiography $[1,9]$.

The peroneal artery is an important vessel for the preparation of the fibular graft and gastrocnemius muscle flap. Preoperative determination of adequacy of the blood supply of the foot after blocking the peroneal artery is also very important. It is very limb-threatening to sacrifice the peroneal artery when the termini of ATA and PTA as the peroneal artery may function as the sole blood supply of the foot $[1,9,12,32-34]$. In the past, blood supply of the foot was determined by the preoperative observation of the pulse of the PTA and dorsalis pedis artery. In recent years, angiography and CTA technology are applied for the evaluation of lower extremity vessels. Although angiography and CTA scans can clearly discern variations in the popliteal artery and its branches, but due to its invasive nature and potential adverse reaction to the contrast agents, their clinical popularity is significantly comprised $[1,9,35,36]$.

Our study had technical limitations. Due to its location, the terminal segment of ATA is not readily resolved on ultrasound. In addition, the ATA is obscured by the tibia when travelling between the tibia and fibula. These factors adversely affect ultrasound examination of the 
full length of the artery, which could explain the higher rate $(3.0 \%)$ of the absence of terminal ATA in this study, comparing to that of $1.6 \%-1.7 \%$ in Kin and Sung's series $[29,37,38]$. Moreover, ultrasound is unable to differentiate absence from the narrowing of the ATA at the lower tibial area. Therefore, ability of ultrasound to probe variation of ATA in the lower tibial area is limited. Other limitations: lack of comparison with other imaging techniques, lack of interobserver and intraobserver agreements.

In conclusion, our results suggest that ultrasound examination of the lower extremity arteries should be applied prior to any intervention in these vessels to prevent undesirable damage. The variant patterns identified in this study have prominent clinical significance.

\section{Conflict of interest: none}

\section{References}

1. Yanik B, Bulbul E, Demirpolat G. Variations of the popliteal artery branching with multidetector CT angiography. Surg Radiol Anat 2015; 37: 223-230.

2. Smith PN, Gelinas J, Kennedy K, Thain L, Rorabeck CH, Bourne RB. Popliteal vessels in knee surgery. A magnetic resonance imaging study. Clin Orthop Relat Res 1999; 367: 158-164.

3. Hirai T, Ohishi H, Kichikawa K, Yoshimura H, Uchida H. Ultrasonographic screening for arterial occlusive disease in the pelvis and lower extremities. Radiat Med 1998; 16 : 411-416.

4. Saadeh FA, el-Karagy SA, Haikal FA. Anterior tibial artery: variation in origin and branching. Surg Radiol Anat 1995; 17: 83-84

5. Vancov V, Marinov G. On the vascularization of lower limb arteries. Folia Morphol (Praha) 1973; 21: 188-190.

6. Mauro MA, Jaques PF, Moore M. The popliteal artery and its branches: embryologic basis of normal and variant anatomy. AJR Am J Roentgenol 1988; 150: 435-437.

7. Stager A, Clement D. Popliteal artery entrapment syndrome. Sports Med 1999; 28: 61-70.

8. Allimant P, Aventin G, Lehn-Hogg M, Mettauer M, Dalcher G. Double popliteal artery of congenital origin. Ann Vasc Surg 1992; 6: 373-377.

9. Calisir C, Simsek S, Tepe M. Variations in the popliteal artery branching in 342 patients studied with peripheral CT angiography using 64-MDCT. Jpn J Radiol 2015; 33: 1320.

10. Longo B, Sorotos M, Nicolotti M, Santanelli di Pompeo F. Retrospective analysis of incidence of peroneal artery hypoplasia in 101 free fibula transfers and new classification of popliteal branch anomalies. Injury 2014; 45: 394398.

11. Hayashi A, Matsumura T, Horiguchi M, et al. The medial plantar flap vascularized by the reverse flow lateral plan- tar artery: a novel variation through the case of aggressive digital papillary adenocarcinoma of the sole. J Reconstr Microsurg 2012; 28: 427-430.

12. Kropman RH, Kiela G, Moll FL, de Vries JP. Variations in anatomy of the popliteal artery and its side branches. Vasc Endovascular Surg 2011; 45: 536-540.

13. Romiti M, Miranda F Jr, Brochado-Neto FC, Kikuchi M, Albers M. Importance of the arteriographic anatomy of the descending genicular artery and sural arteries in patients with atherosclerotic occlusion of the popliteal artery. Vascular 2006; 14: 201-205.

14. Shahid S, Saghir N, Cawley O, Saujani S. A Cadaveric Study of the Branching Pattern and Diameter of the Genicular Arteries: A Focus on the Middle Genicular Artery. J Knee Surg 2015; 28: 417-424.

15. Thiery L. Surgical anatomy of the popliteal fossa. Phlebologie 1986; 39: 57-66.

16. Versluis B, Dremmen MH, Nelemans PJ, et al. MRI of arterial flow reserve in patients with intermittent claudication: feasibility and initial experience. PloS One 2012; 7 : e31514.

17. Versluis B, Backes WH, van Eupen MG, et al. Magnetic resonance imaging in peripheral arterial disease: reproducibility of the assessment of morphological and functional vascular status. Invest Radiol 2011; 46: 11-24.

18. Bradbury AW, Adam DJ, Bell J, et al. Bypass versus Angioplasty in Severe Ischaemia of the Leg (BASIL) trial: A description of the severity and extent of disease using the Bollinger angiogram scoring method and the TransAtlantic Inter-Society Consensus II classification. J Vasc Surg 2010; 51: 32S-42S.

19. Klein AJ, Casserly IP, Messenger JC, Carroll JD, Chen SY. In vivo $3 \mathrm{D}$ modeling of the femoropopliteal artery in human subjects based on $\mathrm{x}$-ray angiography: methodology and validation. Med Phys 2009; 36: 289-310.

20. Pillai J, Levien LJ, Haagensen M, Candy G, Cluver MD, Veller MG. Assessment of the medial head of the gastrocnemius muscle in functional compression of the popliteal artery. J Vasc Surg 2008; 48: 1189-1196.

21. Krnic A, Vucic N, Sucic Z. Duplex scanning compared with intra-arterial angiography in diagnosing peripheral arterial disease: three analytical approaches. Vasa 2006; 35: 86-91.

22. Day CP, Orme R. Popliteal artery branching patterns -- an angiographic study. Clin Radiol 2006; 61: 696-699.

23. Pandharipande PV, Lee VS, Reuss PM, et al. Two-station bolus-chase MR angiography with a stationary table: a simple alternative to automated-table techniques. AJR Am J Roentgenol 2002; 179: 1583-1589.

24. Tai NR, Giudiceandrea A, Salacinski HJ, Seifalian AM, Hamilton G. In vivo femoropopliteal arterial wall compliance in subjects with and without lower limb vascular disease. J Vasc Surg 1999; 30: 936-945.

25. Sutton PA, Dyer JE, Guy A. An unusual combination of sciatic and popliteal artery aneurysms. BMJ Case Rep 2011; 17: 2011

26. Santaolalla V, Bernabe MH, Hipola Ulecia JM, et al. Persistent sciatic artery. Ann Vasc Surg 2010; 24: 691.e7-10. 
27. Shutze WP, Garrett WV, Smith BL. Persistent sciatic artery: collective review and management. Ann Vasc Surg 1993; 7: 303-310

28. Lippert H, Pabst R. Arterial variations in man: classification and frequency: Springer; 1985.

29. Kim D, Orron DE, Skillman JJ. Surgical significance of popliteal arterial variants. A unified angiographic classification. Ann Surg 1989; 210: 776-781.

30. Smith TP, Cragg AH, Berbaum KS, Nakagawa N. Comparison of the efficacy of digital subtraction and film-screen angiography of the lower limb: prospective study in 50 patients. AJR Am J Roentgenol 1992; 158: 431-436.

31. Woo K, Palmer OP, Weaver FA, Rowe VL; Society for Vascular Surgery Vascular Quality Initiative. Use of completion imaging during infrainguinal bypass in the Vascular Quality Initiative. J Vasc Surg 2015; 61: 1258-1263.

32. Wang CY, Chai YM, Wen G, Han P, Cheng L. Superficial peroneal neurocutaneous flap based on an anterior tibial artery perforator for forefoot reconstruction. Ann Plast Surg 2015; 74: 703-707.
33. Lidder S, Masterson S, Dreu M, Clement H, Grechenig S. The risk of injury to the peroneal artery in the posterolateral approach to the distal tibia: a cadaver study. J Orthop Trauma 2014; 28: 534-537.

34. Kishman L, Malin D, Nerone V, Siesel K. Clinical implications of peroneal artery variation. Foot Ankle Spec 2009; 2: 135-139.

35. Foley WD, Stonely T. CT angiography of the lower extremities. Radiol Clin North Am 2010; 48: 367-396.

36. Kramer SC, Gorich J, Aschoff AJ, et al. Diagnostic value of spiral-CT angiography in comparison with digital subtraction angiography before and after peripheral vascular intervention. Angiology 1998; 49: 599-606.

37. Kil SW, Jung GS. Anatomical variations of the popliteal artery and its tibial branches: analysis in 1242 extremities. Cardiovasc Intervent Radiol 2009; 32: 233-240.

38. Szpinda M. Digital-image analysis of the angiographic patterns of the popliteal artery in patients with aorto-iliac occlusive disease (Leriche syndrome). Ann Anat 2006; 188: 377-382. 